OPEN ACCESS

Edited by:

Tao Xu,

Anhui Medical University, China

Reviewed by:

Dongdong Wang,

McMaster University, Canada

Guanlan Xu,

University of Alabama at Birmingham,

United States

*Correspondence: Xiao Wang wangxiao@ahtcm.edu.cn Jihong Fang

fangjihong510@126.com

Specialty section:

This article was submitted to Inflammation Pharmacology,

a section of the journal

Frontiers in Pharmacology

Received: 25 March 2021

Accepted: 10 May 2021

Published: 21 May 2021

Citation:

Miao C, Zhou W, Wang $X$ and Fang $J$ (2021) The Research Progress of Exosomes in Osteoarthritis, With Particular Emphasis on the Mediating Roles of miRNAs and InCRNAs. Front. Pharmacol. 12:685623. doi: $10.3389 /$ fphar.2021.685623

\section{The Research Progress of Exosomes in Osteoarthritis, With Particular Emphasis on the Mediating Roles of miRNAs and IncRNAs}

\author{
Chenggui Miao ${ }^{1,2,3}$, Wanwan Zhou ${ }^{1}$, Xiao Wang $^{4 *}$ and Jihong Fang ${ }^{5,6 *}$ \\ ${ }^{1}$ Department of Pharmacology, School of Integrated Chinese and Western Medicine, Anhui University of Chinese \\ Medicine, Hefei, China, ${ }^{2}$ Department of Pharmacy, School of Life and Health Sciences, Anhui University of Science and \\ Technology, Fengyang, China, ${ }^{3}$ Institute of Prevention and Treatment of Rheumatoid Arthritis of Chinese Medicine, Anhui \\ University of Chinese Medicine, Hefei, China, ${ }^{4}$ Department of Clinical Nursing, School of Nursing, Anhui University of \\ Chinese Medicine, Hefei, China, ${ }^{5}$ Department of Nursing, Anhui Provincial Children's Hospital, Affiliated to Anhui Medical \\ University, Hefei, China, ${ }^{6}$ Department of Orthopedics, Anhui Provincial Children's Hospital, Affiliated to Anhui Medical \\ University, Hefei, China
}

Osteoarthritis $(\mathrm{OA})$ is a kind of degenerative disease, which is caused by many factors such as aging, obesity, strain, trauma, congenital joint abnormalities, joint deformities. Exosomes are mainly derived from the invagination of intracellular lysosomes, which are released into the extracellular matrix after fusion of the outer membrane of multi vesicles with the cell membrane. Exosomes mediate intercellular communication and regulate the biological activity of receptor cells by carrying non-coding RNA, long noncoding RNAs (IncRNAs), microRNAs (miRNAs), proteins and lipids. Evidences show that exosomes are involved in the pathogenesis of OA. In view of the important roles of exosomes in OA, this paper systematically reviewed the roles of exosomes in the pathogenesis of $\mathrm{OA}$, including the roles of exosomes in OA diagnosis, the regulatory mechanisms of exosomes in the pathogenesis, and the intervention roles of exosomes in the treatment of OA. Reviewing the roles of exosomes in OA will help to clarify the pathogenesis of $\mathrm{OA}$ and explore new diagnostic biomarkers and therapeutic targets.

Keywords: exosomes, non-coding RNA, long noncoding RNA, osteoarthritis, microRNA

\footnotetext{
Abbreviations: ADAMTS5, disintegrin and metalloproteinase with thrombospondin motifs 5; ADSCs, adipose-derived stem cells; AFSC, amniotic fluid stem cells; ATF4, transcription factor 4; CAP, chondrocyte-affinity peptide; Col2al, collagen alpha 1; DMM, destabilization of the medial meniscus; E7 exo, E7 peptide; ESC, human embryonic stem cell; HDAC, histone deacetylase; HDAC3, histone deacetylase 3; IPFP, infrapatellar fat pad; KGN, kartogenin; lncRNAs, long noncoding RNAs; MSCs, mesenchymal stem cells; OA, osteoarthritis; pBMSCs, polydactyly bone marrow-derived MSCs; PHCs, primary human chondrocytes; PRP, platelet-rich plasma; SAGA, Spt-Ada-Gcn5 acetyltransferase; SHED, stem cells from human exfoliated deciduous teeth; SMMSC-Exos, synovial mesenchymal stem cells; TLR, toll-like receptor; TMJ-OA, temporomandibular joint osteoarthritis; YAP, yes-associated protein.
} 


\section{INTRODUCTION}

Exosomes are small membrane bubbles (40-150 nm) containing complex RNAs and proteins. Many cells can secrete exosomes in physiological and pathological states. They are mainly derived from the vesicles formed by the collapse of lysosomal particles, which are released into extracellular matrix after fusion of the outer membrane and cell membrane (Zhang and Yu, 2019; Kalluri and LeBleu, 2020).

Almost all types of cells can secrete exosomes, which naturally exist in body fluids, including blood, saliva, urine, cerebrospinal fluid and milk. The precise molecular mechanisms of their secretion and uptake, composition, "carrier" and corresponding functions has just begun to be studied (Meldolesi, 2018). Exosomes are considered as specific vesicles and participate in intercellular communication. There is a growing interest in the study of exosomes, whether to study their functions or to understand how to use them in the development of minimally invasive diagnosis (Mashouri et al., 2019).

When exosomes are secreted from host cells into receptor cells, exosomes can regulate the biological activity of receptor cells by carrying proteins, nucleic acids and lipids (Gonda et al., 2019). Exosome mediated intercellular communication mainly through the following ways. First, exosome membrane proteins can bind to target cell membrane proteins and activate signal pathways in target cells. Second, in the extracellular matrix, exosome membrane proteins can be cleaved by proteases. The cleaved fragments can act as ligands to bind to receptors on the cell membrane, thus activating intracellular signaling pathways. Thirdly, the exosome membrane can fuse directly with the target cell membrane, and non-selectively release proteins, mRNAs, microRNAs (miRNAs) and long noncoding RNAs (lncRNAs) (Familtseva et al., 2019; Jiang et al., 2019).

When exosomes are first discovered, they are considered as a way for cells to excrete waste. Nowadays, with a large number of studies on their biological sources, material composition, transportation, intercellular signal transduction, and distribution in body fluids, exosomes have been found to have a variety of functions (Shan et al., 2019). Exosomes can participate in immune response, antigen presentation, cell migration, cell differentiation, tumor invasion. Studies have shown that exosomes participate in the pathological mechanisms of osteoarthritis (OA), and promote the pathological development of OA (Chen B. Y. et al., 2019; Zhou Q. F. et al., 2020) (Figure 1).

$\mathrm{OA}$ is a kind of degenerative disease, which is caused by many factors such as aging, obesity, strain, trauma, congenital joint abnormalities, joint deformities (Hunter and Bierma-Zeinstra, 2019; Kan et al., 2019). The disease is more common in the

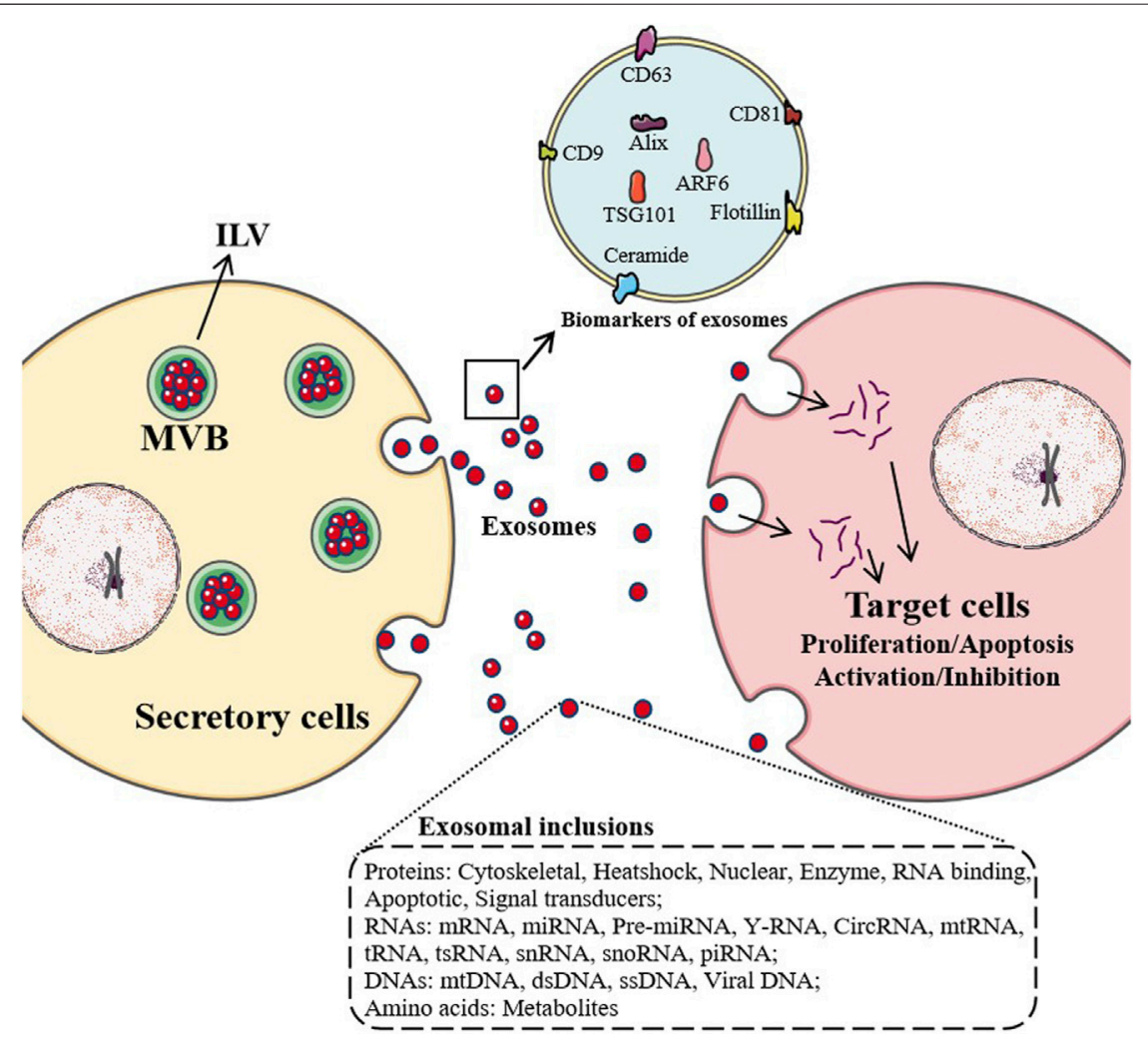

FIGURE 1 | Maturation and secretion mechanisms of exosomes. After most endosomes mature to multivesicular bodies (MVB) or late endosomes, their contents, RNAs, proteins, lipids are packaged as intraluminal vesicles (ILV) in MVB. With the fusion of MVB and cell membrane, ILV are released as exosomes and enter target cells by endocytosis, which affect the physiological and pathological mechanisms of target cells, such as proliferation, apoptosis, activation and inhibition. 
middle-aged and elderly people, and occurs in the weight-bearing joints and joints with more activity (such as cervical spine, lumbar spine, knee joint, hip joint, etc.). Excessive weight bearing or the use of these joints can promote the occurrence of degenerative changes. The clinical manifestations includes joint swelling, joint stiffness, joint movement limitation (Sacitharan, 2019; Abramoff and Caldera, 2020).

The main symptom is joint pain, often rest pain, which is manifested as pain after rest. After a moment of activity, the pain is relieved, but after too much activity, the pain is aggravated (Mandl, 2019). Another symptom is joint stiffness, which often occurs in the morning or after the joint maintains a certain position for a long time in the daytime. Joint swelling can be seen in the affected joints. There is a sense of friction or "click" sound during activity. Muscle atrophy and joint deformity can be found in severe cases (O’Neill and Felson, 2018; Geyer and Schönfeld, 2018).

At present, mesenchymal stem cells (MSCs) are considered to be related to the development of OA. Some people believe that the paracrine of nutritional factors, including exosomes mediated secretion, plays an important role in MSC based OA treatment mechanisms (Colombini et al., 2019; Song et al., 2020). There is evidence that the paracrine of exosomes may play important roles in the repair of joint tissue. Exosomes isolated from various stem cells contribute to tissue regeneration of heart, limbs, skin and other tissues, and exosomes derived from MSCs may inhibit the pathological development of OA (Mianehsaz et al., 2019).

In this work, we reviewed the role of exosomes in the pathogenesis of OA, which will help to clarify the pathogenesis of $\mathrm{OA}$ and explore new diagnostic biomarkers and therapeutic targets.

\section{CIRCULATING EXOSOMES AS DIAGNOSTIC BIOMARKERS OF OSTEOARTHRITIS \\ Exosomes in Peripheral Blood}

Exosomes may play important roles in OA diagnosis, and the miRNAs, lncRNAs and other inclusions in exosomes may be new biomarkers for OA diagnosis (Otahal et al., 2020). In OA, miR$193 \mathrm{~b}-3 \mathrm{p}$ regulates the chondrogenesis and chondrocyte metabolism by targeting the histone deacetylase 3 (HDAC3). The expression of miR-193b-3p increases in chondrogenic and hypertrophic human MSCs, but decreases in degenerative cartilage. The level of plasma exosomal miR-193b-3p in OA patients is significantly lower than that in control group, suggesting that the exosomal miR-193b-3p may be a new diagnostic marker for OA (Meng et al., 2018).

Plasma exosomes of OA patients can induce OA chondrocytes to express cartilage genes and inhibit the release of inflammatory cytokines. This highlights the potential of plasma exosome inclusions as regulators of extracellular matrix metabolism and inflammation, and may be candidates for a new approach of cellfree therapy and diagnosis of OA (Chang et al., 2018).

Exosomes in plasma of OA patients have been found to have new potential in relieving knee osteoarthritis. The mechanisms may be that exosome inclusions promote the chondrocyte proliferation and inhibit the chondrocyte apoptosis through the $\mathrm{Wnt} / \beta$-catenin signaling pathway. It also suggests the feasibility of exosome inclusions as diagnostic markers for OA (Yuan et al., 2016). In OA chondrocytes treated by IL-1 $\beta$, plateletrich plasma (PRP) exosomes can inhibit the release of TNF- $\alpha$, promote the proliferation of OA chondrocytes, and significantly reduce the apoptosis rate of OA chondrocytes. PRP exosomes, as carriers containing PRP derived growth factors, provide a new way for the diagnosis of OA (He et al., 2020).

The levels of lncRNA PVT1 and high mobility groupprotein B1 (HMGB1) are up-regulated, while the level of miR-93-5p is down-regulated in serum and LPS induced C28/I2 cells. PVT1 deletion can reverse the decrease of cell viability, increase of apoptosis and inflammation induced by LPS in C28/I2 cells. PVT1 regulates the expression of HMGB1 through miR-93-5p. Inhibition of miR-93-5p can eliminate the apoptosis, inflammatory response and collagen degradation of C28/I2 cells mediated by PVT1 silencing. The increase of HMGB1 reverses the up-regulation of miR-93-5p mediated apoptosis and inflammation of C28/I2 cells. In addition, PVT1 regulates the TLR4/NF- $\kappa B$ pathway through miR-93-5p/HMGB1 axis. Obviously, PVT1 gene knockout by exosomes can inhibit the pathological development of OA through miR-93-5p mediated HMGB1/TLR4/NF- $\mathrm{kB}$ pathway, and these exosomal inclusions may be new diagnostic markers for OA (Xue et al., 2019; Meng et al., 2020; Sun et al., 2020).

\section{Exosomes in Synovial Fluid}

There are gender differences in exosome proteins in synovial fluid of patients with OA (Kim et al., 2020). Studies have shown that exosome-derived miRNAs in synovial fluid of OA patients were changed, and these changes were gender specific. For example, female OA specific miRNAs targeted the estrogen responsive tolllike receptor (TLR) signaling pathway. After OA derived exosomes treatment, the expression of anabolic genes decreased, catabolic genes were up-regulated, and the expression of inflammatory genes also increased significantly (Kolhe et al., 2017).

In the synovial fluid exosomes of female OA, the levels of haptoglobin, orosomucoid and ceruloplasmin are up-regulated, while the level of apolipoprotein is down-regulated. In the synovial fluid exosomes of male OA, the levels of $\beta-2$ glycoprotein and complement component five protein are significantly up-regulated, while the level of Spt-Ada-Gcn5 acetyltransferase (SAGA)-related factor 29 is down-regulated. There are gender differences in synovial fluid exosome protein content in OA patients, which indicates that these differentially expressed proteins may be new diagnostic markers for OA (Kolhe et al., 2020).

Synovial fluid contains various cytokines, and most of them are not only in free form, but also enriched in exosomes (Carlson et al., 2018). Compared with the cytokine spectrum of synovial fluid, the exosomes of patients with end-stage OA have more cytokine content, especially chemokines. Synovial fluid derived exosomes recruit inflammatory cells, inhibit cartilage proliferation and promote joint degeneration. Synovial fluid 


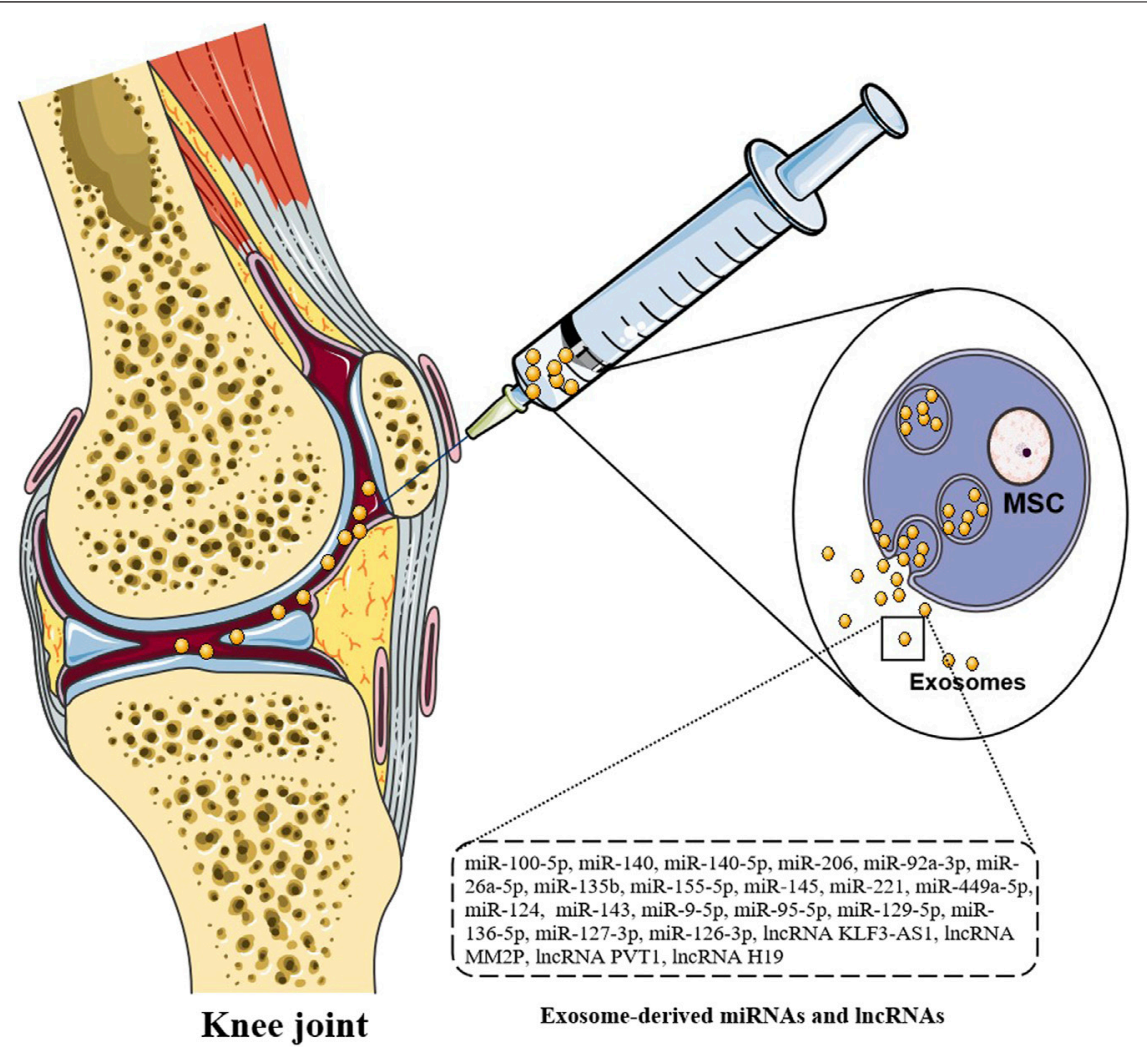

FIGURE 2 | Injection therapy of exosomes. Direct injection of exosomes carrying miRNAs and IncRNAs can significantly down-regulate arthritis score, inhibit FLS proliferation and invasion, and reduce inflammatory response and joint damage.

microenvironment and exosomes mediated intercellular communication provide a new perspective for OA pathological research, and these exosomal cytokines may be new diagnostic biomarkers for OA (Gao et al., 2020).

Exosomal lncRNAs in synovial fluid are valuable in the differential diagnosis of early and late-stage OA. For example, the levels of exosomal lncRNAs in early OA and late-stage OA synovial fluid are significantly higher than that in control group. The expression of lncRNA PCGEM1 in patients with late-stage $\mathrm{OA}$ is significantly higher than that in patients with early $\mathrm{OA}$, and the expression of PCGEM1 in early OA is significantly higher than that in control group. Exosomal PCGEM1 may be a powerful indicator to differentiate early $\mathrm{OA}$ from late-stage OA (Zhao and Xu, 2018).

\section{EXOSOMES IN OSTEOARTHRITIS PATHOGENESIS}

Exosomes from various tissues play important roles in the pathogenesis of OA. IL-1 $\beta$ induced MSCs exosomes have obvious anti-inflammatory activity in OA sw982 cells. The roles of IL- $1 \beta$ in inducing MSC-derived exosomes is mediated by miR-147b, leading to the inhibition of NF- $\kappa B$ pathway (Kim et al., 2021). By comparing the expression of miRNAs in the exosomes of human bone marrow MSCs with that without cartilage induction, 141 differentially expressed miRNAs are found, including 35 up-regulated miRNAs, such as miR-92a, miR-193a-5p, miR-320c, miR-1246, miR-1290, 106 down -regulated miRNAs, such as miR-377-3p and miR-6891-5p. $\mathrm{MiR}-320 \mathrm{c}$ in the induced exosomes promotes the proliferation of OA chondrocytes and down-regulates the MMP13 expression more than that in control group. These miRNAs can induce cartilage and may play important roles in cartilage regeneration and the final treatment of OA (Sun et al., 2019) (Figure 2).

\section{Exosomes Derived From Synovial Mesenchymal Stem Cells}

Exosomes derived from OA synovial MSCs with miR-140-5p overexpression are effective for OA treatment. Wnt5a and wnt5b in exosomes can activate the Yes-associated protein (YAP) through the Wnt signaling pathway and promote the proliferation and migration of chondrocytes. The side effect is significantly reduction of the ECM secretion, and the high expression of miR-140-5p blocks this side effect. In vivo, Exosomes derived from miR-140-5p-overexpressing human synovial MSCs successfully prevent the OA in model rats (Tao et al., 2017). MiR-129-5p in human synovial MSC exosomes attenuates the IL- $1 \beta$ induced OA by targeting the HMGB1. In OA patients and IL-1 $\beta$ induced chondrocytes, miR-129-5p is decreased, while HMGB1 is significantly up-regulated. 
MiR-129-5p targets the $3^{\prime}$ UTR of HMGB1 and inhibits the upregulation of HMGB1. Exosomes rich in miR-129-5p can significantly reduce the inflammatory response and apoptosis of chondrocytes, while exosomes lacking miR-129-5p increases the inflammatory response and apoptosis of chondrocytes (Qiu et al., 2021).

Synovial fluid derived MSCs transplantation is an effective method to treat OA cartilage degeneration. kartogenin $(\mathrm{KGN})$ is a small molecule that can induce MSCs to differentiate into chondrocytes in vitro and in vivo. It controls the chondrogenic differentiation of transplanted MSCs. However, the poor water solubility of KGN limits its clinical application. The exosomes containing KGN can effectively solve this technical problem. MSCs binding peptide E7 is fused with exosome membrane protein Lamp $2 \mathrm{~b}$ to produce exosomes with surface display E7 peptide (E7 exo). The E7 exo containing KGN could effectively enter MSCs, and the degree of cartilage differentiation is higher than that of KGN alone or without E7 exo (Xu et al., 2021). The E7 exo containing KGN pretreated bone marrow MSCs have higher cartilage repair efficiency, stronger cartilage matrix formation and less degradation than those from bone marrow MSCs (Liu et al., 2020).

In exosomes derived from synovial fibroblasts, overexpressed miR-126-3p inhibits the chondrocyte inflammation and cartilage degradation in OA model rats, which may have certain therapeutic value for OA patients (Zhou et al., 2021). MiR$26 a-5 p$ is low expressed in OA patients and synovial fibroblasts treated with IL-1 $\beta$, while PTGS2 is high expressed. PTGS2 is a direct target of miR-26a-5p. Overexpression of miR$26 a-5 p$ alleviates the injury of synovial fibroblasts by inhibiting the PTGS2. Obviously, overexpression of miR-26a-5p inhibits the injury of synovial fibroblasts in OA through the PTGS2, which is of great significance for the treatment of OA (Jin et al., 2020b).

Exosomal lncRNA H19 in synovial fibroblasts attenuates the progression of OA through miR-106b-5p/TIMP2 axis. Cartilage repair mediated by exosomes is characterized by increased cell viability and migration as well as reduced matrix degradation. In the process of exosomes mediated cartilage repair, the enhancement of cell proliferation and migration is related to the regulation of miR-106b-5p/TIMP2 axis. Transfection of miR-106-5p mimics in chondrocytes significantly reduces the cell proliferation and migration, promotes the matrix degradation, increases the expression of MMP13 and ADAMT5, and decreases the expression of COL2A1 and ACAN in chondrocytes. TIMP2 is directly regulated by miR106-5p. This suggests that H19 may promotes the chondrocyte proliferation and migration and inhibits the degradation of $\mathrm{OA}$ matrix by targeting miR-106b-5p/TIMP2 axis in OA pathogenesis (Tan et al., 2020).

\section{Exosomes Derived From Bone Marrow Mesenchymal Stem Cells}

Exosomal lncRNA KLF3-AS1 from human bone marrow MSCs can be used as an effective therapeutic molecule for OA patients. KLF3-AS1 is significantly enriched in the exosomes of MSCs. The exosomal KLF3-AS1 inhibits the apoptosis of chondrocytes and improves the cartilage injury induced by IL-1 $\beta$. KLF3-AS1 may be a potential therapeutic target for OA (Liu et al., 2018b).

In OA model mice induced by collagenase, bone marrow MSC-derived exosomes increase the chondrogenic genes type II collagen alpha 1 (Col2a1) and aggrecan, decrease the markers of chondrocyte hypertrophy MMP-13 and runt-related transcription factor 2 (Runx2), and attenuate the IL-1 $\beta$ induced inhibition of chondrocyte proliferation. Exosomes derived from KLF3-AS1-overexpressing-MSCs ameliorate the IL-1 $\beta$ induced chondrocyte injury. Interestingly, KLF3-AS1 promotes the GIT1 expression by adsorbing the miR-206. Thus bone marrow MSC-derived exosomes promote the osteoarthritis chondrocyte proliferation and inhibit the apoptosis through the KLF3-AS1/miR-206/GIT1 axis (Liu et al., 2018a).

MiR-127-3p is enriched in bone marrow MSC-derived exosomes, and bone marrow MSC-derived exosomes inhibit the IL- $1 \beta$ induced chondrocyte injury. MiR-127-3p can inhibit the CDH11 of chondrocytes, thus blocking the activation of Wnt/ $\beta$-catenin pathway and alleviating the damage of $\mathrm{OA}$ chondrocytes (Dong et al., 2021).

Mao et al. (2018b) investigated the expression of miR-92a-3p in human bone marrow MSC chondrogenesis model and OA primary human chondrocytes (PHCs) in vitro. Interestingly, the expression of miR-92a-3p increased in MSC chondrocytes and decreased in OA chondrocytes. MSC-miR-92a-3p-exos promoted the cartilage proliferation and matrix gene expression of MSCs and PHCs, while MSC-anti-miR-92a-3pexos inhibited the chondrocyte differentiation and cartilage matrix synthesis by enhancing the Wnt5a expression. MiR$92 \mathrm{a}-3 \mathrm{p}$ regulates the cartilage development and homeostasis by directly targeting the $\mathrm{Wnt} 5 \mathrm{a}$, suggesting its important roles in OA pathology.

After IL- $1 \beta$ is added to rabbit bone marrow MSCs and chondrocytes cultured in vitro, chondrocyte viability decreases, cell apoptosis and mitochondrial membrane potential changes significantly. However, these changes disappeared after adding bone marrow MSC-derived exosomes. Compared with IL-1 $\beta$ group, the exosomes of bone marrow MSCs inhibit the phosphorylation of p38 and ERK, and promote the phosphorylation of Akt. These findings suggest that bone marrow MSC-derived exosomes inhibit the chondrocyte apoptosis through p38, ERK and Akt pathways (Qi et al., 2019).

MSCderived exosomes inhibit the pathogenesis of OA by inhibiting syndecan-1. Injection of exosomes containing miR9-5p can alleviate inflammation and OA like injury, downregulate the levels of inflammatory factors, alleviate the oxidative stress injury, and reduce the levels of OCN, MMP13, comp and AKP. Syndecan-1 is the target of miR-9-5p. Upregulation of syndecan-1 leads to aggravation of inflammation and OA like injury. The exosomal miR-9-5p derived from bone marrow MSC has anti-inflammatory and cartilage protective effects on OA by regulating the syndecan-1 (Jin et al., 2020a).

The expression of ELF3 increases and the expression of miR$136-5 p$ decreases in traumatic OA cartilage. MiR-136-5p is confirmed to target the ELF3 and down-regulates its expression. After chondrocytes internalizes exosomes, the 
expression of ELF3 decreases. The exosomal miR-136-5p, derived from bone marrow MSCs, can promote the chondrocyte migration in vitro and inhibits the cartilage degeneration in vivo, thereby inhibiting the pathological changes of OA (Chen et al., 2020).

The stimulating effects of exosomes isolated from osteoblasts of coxarthrosis on bone marrow MSCs are mainly manifested in the catabolism and osteogenic differentiation. Interestingly, this has nothing to do with donor pathology, reflecting the influence of exosomes on tissue microenvironment and cell metabolism in coxarthrosis (Niedermair et al., 2020).

\section{Exosomes Derived From Primary Chondrocytes}

The exosomes of primary chondrocytes may play a role in the treatment of OA. Zheng et al. (2019) isolated exosomes from primary chondrocytes cultured in normal and IL- $1 \beta$ induced inflammatory environment, and found that there were more mitochondrial proteins in exosomes of chondrocytes in normal group. Intra-articular injection of exosomes of chondrocytes in normal group successfully prevented the development of OA. Studies have shown that chondrocyte exosomes restored the mitochondrial dysfunction and promoted the macrophages to differentiate into M2 phenotypes.

Drug delivery is the key to the successful clinical application of nucleic acid drugs, and exosomal miRNAs in the treatment of OA has brought a new perspective to the treatment of OA. MiR-140 is not only significant in promoting the cartilage formation and inhibiting the degeneration, but also plays an important role in cartilage development (Duan et al., 2020). Liang et al. (2020) fused chondrocyte-affinity peptide (CAP) with lysosome associated membrane glycoprotein $2 \mathrm{~b}$ on the surface of the exosomes to obtain cap exosomes. This exosomes could effectively encapsulate miR-140, specifically enter chondrocytes, and transport miR-140 to chondrocytes. Studies have shown that cap exosomes could also transfer miR-140 to the deep cartilage region through the dense mesochondral membrane, inhibited the cartilage degradation protease, and suppress the progress of OA.

MiR-95-5p regulates the chondrogenesis and cartilage degradation through the histone deacetylase (HDAC) $2 / 8$. MiR-95-5p overexpression of primary chondrocyte derived exosomes may be effective in the treatment of OA. HDAC 2/8 is up-regulated in $\mathrm{OA}$ tissues and exosomes secreted by chondrocytes, and mediates the expression of specific genes in chondrocytes. MiR-95-5p directly acts on the $3^{\prime}$ UTR of HDAC 2/ 8 to promote the cartilage formation and prevent $\mathrm{OA}$ by directly targeting the HDAC 2/8 (Mao et al., 2018a).

Transcription factor 4 (ATF4) plays an important role in chondrocyte proliferation and bone formation. It has been proved that the serum derived exosomes of OA mice has therapeutic effects on OA model mice (Chen D. et al., 2019). Studies have shown that intra-articular injection of ATF4-OAExosomes can reduce the degeneration, damage and inflammatory reaction of articular cartilage in OA model mice, and partially restore the autophagy function of knee cartilage.
Furthermore, ATF4-OA-Exosomes promote the autophagy and inhibit the apoptosis in TNF- $\alpha$ or tunicamycin treated chondrocytes (Wang Y. et al., 2021).

\section{Exosomes Derived From Human Embryonic Stem Cells}

The exosomes derived from human ESC-induced MSCs (ESCMSCs) are involved in the therapeutic mechanisms of alleviating OA. In destabilization of the medial meniscus (DMM) model mice, intra-articular injection of ESC-MSCs reduces the cartilage destruction and matrix degradation in DMM model, which is mediated by ESC-MSCs derived exosomes. In the presence of IL$1 \beta$, these exosomes maintain the chondrocyte phenotype by increasing the type II collagen synthesis and decreasing the ADAMTS5 expression. Immunocytochemistry shows that the exosomes are colocalized with type II collagen positive chondrocytes. This provides a new target for the development of OA drugs and drug delivery systems (Wang et al., 2017).

\section{Exosomes Derived From Chondrogenic Progenitor Cells}

Chondrogenic progenitor cells have high self-renewal ability and chondrogenic potential. Wang et al. (2020) found that intraarticular injection of exosomes secreted by chondrogenic progenitor cells in $\mathrm{MRL} / \mathrm{MpJ}$ superhealer mice promoted the repair of articular cartilage injury in mice. By comparing the miRNA expression profiles of control CBA (CBA EVs) and MRL/ MPJ mouse chondroblasts, the differentially expressed exosomal miRNAs were involved in a variety of biological processes. Among them, 80 miRNAs were significantly up-regulated and 100 were down-regulated, and 20 disordered miRNAs linked OA repair through the AMPK signaling, autophagy regulation and insulin signaling. The mechanisms of exosomes involved in OA may be more related to miRNAs (Toh et al., 2017).

\section{Exosomes Derived From Vascular Endothelial Cells}

Exosomes from vascular endothelial cells have been proved to be involved in the pathogenesis of many diseases, and their roles in the OA pathogenesis have also been confirmed. Exosomes derived from vascular endothelial cells promote the pathological development of OA by inducing the chondrocyte apoptosis. These exosomes can inhibit the autophagy and p21 expression, reduce the ability of chondrocytes to resist the oxidative stress, increase the content of ROS and induce the apoptosis. Exosomes derived from vascular endothelial cells promote the progress of $\mathrm{OA}$ and provide new ideas for the diagnosis and treatment of OA (Yang et al., 2021).

\section{Exosomes Derived From Adipose-Derived Stem Cells}

ADSCs are candidate cells for anti-inflammatory and cytoprotective effects on cartilage. Exosomes mediate the 
paracrine effect of ADSCs and down-regulate the aging characteristics of OA osteoblasts (Tofiño-Vian et al., 2017). ADSCs promote the chondrogenesis and inhibit the inflammation. Patients with OA are usually associated with obesity and chronic inflammation. Exosomes isolated from ADSCs down-regulate the expression of IL- $6, N F-\kappa B$ and TNF- $\alpha$, and up-regulate the expression of IL-10. Exosome therapy can protect the articular chondrocytes from $\mathrm{H} 2 \mathrm{O} 2$ induced apoptosis. In addition, exosome therapy promotes the chondrogenesis of periosteal cells and increases the level of chondrogenic markers, including type II collagen and $\beta$-catenin. Wnt signaling pathway may be its downstream signaling pathway. The periosteal cells with exosomes show high levels of miR-145 and miR-221, and the miR-145 and miR-221 are related to the enhancement of periosteal cells and chondrogenic potential, respectively (Zhao et al., 2020).

For exosomes derived from MSCs of infrapatellar fat pad (IPFP), miR-100-5p-abundant exosomes protect the articular cartilage and improve the gait abnormality by inhibiting the mTOR signal of OA. IPFP MSCs can produce a large number of exosomes, which show typical morphological characteristics of exosomes. IPFP MSC-derived exosomes can reduce the severity of $\mathrm{OA}$, inhibit the apoptosis, promote the matrix synthesis and reduce the expression of catabolic factors. These exosomes can significantly enhance the autophagy level of chondrocytes through the mTOR inhibition. The detection of luciferase reporter gene shows that mir-100-5p combines with the mTOR's $3^{\prime} \mathrm{UTR}$, which reverses the mTOR signal pathway. It is important that intra-articular injection of antagormir-100-5p significantly reduces the protective effects of MSC-derived exosomes on articular cartilage. These IPFP MSCs are expected to be a potential therapy for OA (Clockaerts et al., 2010; Wu et al., 2019).

\section{Exosomes Derived From Human Dental Pulp Stem Cells}

Exosomes of human DPSCs can inhibit the chondrocyte apoptosis in OA model rats. After transfection of DPSCs with miR-140-5p mimics, the exosomal miR-140-5p increased significantly. In IL- $1 \beta$ treated human chondrocytes, DPSCderived exosomes promote the expression of chondrocyte related mRNAs, including aggrecan, Col2a1 and Sox9. Exosomes containing miR-140-5p significantly enhance this phenomenon. MiR-140-5p rich exosomes derived from DPSCs may play an anti-apoptotic role by regulating the expression of apoptosis related proteins. The exosomes of DPSCs may be a potential strategy for the treatment of OA (Lin et al., 2021).

Exosomes derived from stem cells of human exfoliated deciduous teeth (SHED) have a certain therapeutic effect on temporomandibular arthritis. MiR-100-5p is enriched in these exsomes. SHED exosomes inhibit the expression of IL-6, IL-8, MMP1, MMP3, MMP9, MMP13, disintegrin and metalloproteinase with thrombospondin motifs 5 (ADAMTS5). The chondrocytes treated with miR-100 mimics show low expression of the MMP1, MMP9, MMP13, ADAMTS5 and mTOR. On the contrary, miR-100 inhibition up-regulates these targets. Furthermore, miR-100-5p directly targets the $3^{\prime} \mathrm{UTR}$ of mTOR, and inhibits the expression of mTOR (Luo et al., 2019).

\section{Exosomes Derived From Monocyte Derived Cells}

LncRNA MM2P and exosomes mediate the Sox9 transfer from monocyte derived cells to primary chondrocytes. Treatment of RAW264.7 mouse macrophages and mouse bone marrowderived macrophages with IL-4 or IL-13 up-regulate the expression of MM2P. MM2P blocks the SHP2 mediated dephosphorylation of STAT3 at Try705 and interacts with RNA binding protein FUS. In turn, p-STAT3 increases the Sox9 gene expression. These cells release the Sox 9 mRNA and exosomes containing proteins. The supernatant of these cells can promote the differentiation of primary chondrocytes, that is, upregulates the expression of the Colla2 and Acan genes, and promotes the secretion of extracellular matrix components. These effects are mediated by Sox 9 mRNA and protein delivered to chondrocytes by exosomes. MM2P and its exosomes may be new therapeutic and diagnostic targets for OA (Bai et al., 2020).

\section{Exosomes Derived From Amniotic Fluid Stem Cells}

The exosomes secreted by AFSCs have a certain effect on the treatment of OA. AFSCs can secrete exosomes with growth factors and immune regulatory molecules, which can prevent tissue degradation and induce the cartilage repair. Compared with the control group, the exosomes treated model animals show stronger pain tolerance and improved the histological score. Exosomes containing TGF- $\beta$ can induce the cartilage recovery, which has better surface regularity and hyaline cartilage characteristics, and is positively correlated with the content of TGF- $\beta$. It is easier to detect macrophage markers in exosomes treated joints, which suggests that AFSC exosomes can regulate the macrophage polarization (Beretti et al., 2018; Zavatti et al., 2020).

\section{Exosomes Derived From Polydactyly Bone Mesenchymal Stem Cells}

Zhou X. et al. (2020) obtained a special kind of MSCs from the bone marrow of patients with polydactyly, and found that polydactyly bone marrow-derived MSCs (pBMSCs) played certain roles in the pathological mechanisms of OA. It was important that pBMSCs have stronger ability to differentiate into chondrocytes than BMSCs. Exosomes secreted by pBMSCs stimulated the migration and proliferation of chondrocytes. The expression of BMP4 in pBMSCs was significantly higher than that in BMSCs, and the pBMSCs regulate chondrocyte formation through the BMP4 signal. 


\section{Exosomes Derived From Mesenchymal Stem Cells of Temporomandibular Joint Osteoarthritis}

In the immunocompetent model rats of TMJ-OA, MSCderived exosomes play a key role in inflammatory response, injury behavior, condylar cartilage and subchondral bone healing. Exosomes mediated TMJ repair of OA is characterized by early inhibition of pain and degeneration, followed by reduced inflammation and sustained proliferation. MSC-derived exosomes gradually improve the matrix expression and subchondral bone structure, and promote the overall joint repair and regeneration. MSC-derived exosomes enhance the synthesis of s-GAG synthesis blocked by IL-1 $\beta$, and inhibit the production of nitric oxide and MMP13 induced by IL-1 $\beta$. Interestingly, adenosine receptor activation, Akt, ERK and AMPK phosphorylation inhibitors can partially eliminate these effects. Obviously, MSC-derived exosomes promote the repair and regeneration of TMJ in OA through a well coordinated mechanism (Cui et al., 2017; Zhang et al., 2019).

\section{Exosomes Derived From Mesenchymal Stem Cells of Lumbar Facet Joint Osteoarthritis}

LFJ OA is one of the common causes of low back pain. In the try of mouse bone marrow MSC-derived exosome treatment, exosomes block the abnormal CGRP positive nerves and abnormal H-type angiogenesis in the LFJ subchondral bone, alleviating the low back pain. Bone marrow MSC-derived exosomes reduce the cartilage degeneration, inhibit the expression of tartrate resistant acid phosphatase, reduce the activation of RANKL-RANK-TRAF6 signal, and promote the subchondral bone reconstruction. Bone marrow MSC-derived exosomes have significant protective effects on patients with LFJ$\mathrm{OA}$, which may be a potential choice for the treatment of LFJ-OA (Li et al., 2020).

TGF- $\beta 1$, transforming growth factor $\beta 1$, regulates the proliferation of chondrocytes through MSC-derived exosomes. In the OA model, TGF- $\beta 1$ stimulation enhances the expression of miR-135b in the MSC-derived exosomes and increases the survival rate of $\mathrm{C} 5.18$ cells. Interestingly, there is a negative regulatory relationship between $\mathrm{miR}-135 \mathrm{~b}$ and $\mathrm{Sp} 1$. The combination of TGF- $\beta 1$ and miR-135b inhibitor lead to the decrease of C5.18 cell activity. Obviously, TGF- $\beta 1$ inhibits the SP1 through miR-135b derived from MSC-derived exosomes, and promotes the chondrocyte proliferation, and then promotes the cartilage repair (Wang et al., 2018).

\section{Effects of Exosomes on Macrophages in Osteoarthritis}

Macrophages are derived from mononuclear cells in the blood after penetrating blood vessels. After entering connective tissue, the volume of monocytes increases, the endoplasmic reticulum and mitochondria proliferates, the lysosomes increases and phagocytic function enhances (Meng et al., 2019; Barrett, 2020). Macrophages have a series of continuous functional states. M1 and M2 macrophages are the two extremes of this continuous state. M1 macrophages participate in the positive immune response and play a role in immune surveillance by secreting pro-inflammatory cytokines and chemokines, and presenting antigens. M2 macrophages only have weak antigen presenting ability, and downregulate the immune response by secreting inhibitory cytokines such as IL-10 or TGF-b, which play an important role in immune regulation. Macrophages play important regulatory roles in $\mathrm{OA}$ tissue repair, inflammatory response and chondrocyte proliferation (Wang S. et al., 2019; Russell et al., 2019).

Exosomes of OA chondrocytes promote the production of IL$1 \beta$ in macrophages. These exosomes inhibit the LPS induced autophagy by inhibiting the expression of ATG4B through miR$449 \mathrm{a}-5 \mathrm{p}$. The decrease of autophagy leads to the production of mitochondria, which further enhances the activation of inflammatory bodies and the subsequent production of IL-1 $\beta$. This provides a new perspective for understanding the activation of synovial macrophages and OA pathogenesis in patients with OA (Ni et al., 2019).

MiR-135b is highly expressed in MSC-derived exosomes stimulated by TGF- $\beta 1$. MiR-135b mimics induce the M2 polarization of synovial macrophases. The effects of miR-135b and TGF- $\beta 1$-stimulated exosomes on the polarization of M2 synovial macrophases will be reversed by the increase of MAPK6. In conclusion, MSC-derived exosomal miR-135b promotes the polarization of M2 synovial macrophages by targeting the MAPK6, thus alleviating the cartilage damage and providing a new target for the treatment of OA (Wang and $\mathrm{Xu}, 2021$ ).

$\mathrm{OA}$ is a chronic degenerative disease, which leads to limited activity and even disability. Exosomes derived from bone marrow MSCs can delay the progression of OA (Zhao et al., 2018). Exosomes reduce the cartilage damage and synovial macrophage infiltration, inhibit the M1 macrophage production and promote the M2 macrophage production. Exosomes reduce the expression of pro-inflammatory cytokines IL- $1 \beta$, IL- 6 and TNF- $\alpha$ in synovial fluid, and increase the release of anti-inflammatory IL-10. It is important that macrophages treated by exosomes maintain chondrogenic properties of chondrocytes. Obviously, bone marrow MSCs-derived exosomes alleviate the $\mathrm{OA}$ by promoting the phenotype transformation of synovial macrophages from M1 to M2 (Zhang et al., 2020).

The synovial exosomes stimulate the release of many inflammatory cytokines, chemokines and metalloproteinases by macrophages in OA, but do not affect the expression of CD80 and CD86 costimulator molecules. The purified exosomes has obvious functional activity in stimulating the release of proinflammatory factors by M1 macrophages (Domenis et al., 2017) (Table 1). 
TABLE 1 | Exosomes reported in the pathogenesis of OA.

\begin{tabular}{|c|c|c|c|c|c|}
\hline $\begin{array}{l}\text { Classification of } \\
\text { exosomes }\end{array}$ & $\begin{array}{l}\text { Exosomal } \\
\text { inclusions }\end{array}$ & $\begin{array}{l}\text { Origin of } \\
\text { exosomes }\end{array}$ & Regulatory roles & Targets & References \\
\hline $\begin{array}{l}\text { Monocyte-derived } \\
\text { exosomes }\end{array}$ & LncRNA MM2P & $\begin{array}{l}\text { RAW264.7 mouse macrophages } \\
\text { and mouse bone marrow-derived } \\
\text { macrophages }\end{array}$ & $\begin{array}{l}\text { Promotes the chondrocyte differentiation } \\
\text { and functions }\end{array}$ & Sox9 & $\begin{array}{l}\text { Bai et al. } \\
(2020)\end{array}$ \\
\hline MSC-derived exosomes & MiR-136-5p & $\begin{array}{l}\text { OA chondrocytes and mouse } \\
\text { model of post-traumatic OA }\end{array}$ & $\begin{array}{l}\text { Inhibits the chondrocyte degeneration in } \\
\text { traumatic osteoarthritis }\end{array}$ & ELF3 & $\begin{array}{l}\text { Chen et al. } \\
(2020)\end{array}$ \\
\hline MSC-derived exosomes & MiR-127-3p & $\begin{array}{l}\text { Bone marrow MSCs and primary } \\
\text { chondrocytes of model rats }\end{array}$ & $\begin{array}{l}\text { Inhibits the } \mathrm{CDH} 11 \text { in chondrocytes and } \\
\text { relieving the chondrocyte damage in OA. }\end{array}$ & $\begin{array}{l}\mathrm{CDH} 11 \text {-mediated } \\
\text { wnt } / \beta \text {-catenin } \\
\text { pathway }\end{array}$ & $\begin{array}{l}\text { Dong et al. } \\
(2021)\end{array}$ \\
\hline MSC-derived exosomes & MiR-9-5p & $\begin{array}{l}\text { Rat model induced by anterior } \\
\text { cruciate ligament/medial collateral } \\
\text { ligament transection }\end{array}$ & $\begin{array}{l}\text { Has anti-inflammatory and cartilage } \\
\text { protective effects on } O A\end{array}$ & Syndecan-1 & $\begin{array}{l}\text { Jin et al. } \\
\text { (2020a) }\end{array}$ \\
\hline MSC-derived exosomes & MiR-26a-5p & $\begin{array}{l}\text { MSC and synovial fibroblasts of OA } \\
\text { model rats }\end{array}$ & $\begin{array}{l}\text { Retards the damage of synovial } \\
\text { fibroblasts in vitro and alleviates the OA } \\
\text { damage }\end{array}$ & PTGS2 & $\begin{array}{l}\text { Jin et al. } \\
\text { (2020b) }\end{array}$ \\
\hline CAP exosomes & MiR-140 & Chondrocytes of OA patients & $\begin{array}{l}\text { Inhibits the cartilage-degrading } \\
\text { proteases, and alleviates the OA } \\
\text { progression in mode rats }\end{array}$ & $\begin{array}{l}\text { Cartilage-degrading } \\
\text { proteases }\end{array}$ & $\begin{array}{l}\text { Liang et al. } \\
(2020)\end{array}$ \\
\hline $\begin{array}{l}\text { Exosomes derived from mir- } \\
\text { 140-5p-overexpressing } \\
\text { human DPSCs }\end{array}$ & MiR-140-5p & $\begin{array}{l}\mathrm{IL}-1 \beta \text { treated human chondrocytes } \\
\text { and OA model rats }\end{array}$ & $\begin{array}{l}\text { Inhibits the chondrocyte apoptosis and } \\
\text { improves the knee joint conditions in rat } \\
\text { model }\end{array}$ & $\begin{array}{l}\text { Chondrocyte } \\
\text { apoptosis genes }\end{array}$ & $\begin{array}{l}\text { Lin et al. } \\
(2021)\end{array}$ \\
\hline MSC-derived exosomes & $\begin{array}{l}\text { LncRNA KLF3- } \\
\text { AS1, miR-206 }\end{array}$ & $\begin{array}{l}\text { IL-1 } \beta \text {-induced OA chondrocytes } \\
\text { and collagenase-induced mouse } \\
\text { OA model }\end{array}$ & $\begin{array}{l}\text { Promotes the proliferation and inhibits } \\
\text { apoptosis of chondrocytes }\end{array}$ & $\begin{array}{l}\text { KLF3-AS1/miR- } \\
\text { 206/GIT1 axis }\end{array}$ & $\begin{array}{l}\text { Liu et al. } \\
\text { (2018a) }\end{array}$ \\
\hline MSC-derived exosomes & $\begin{array}{l}\text { LncRNA } \\
\text { KLF3-AS1 }\end{array}$ & $\begin{array}{l}\mathrm{IL}-1 \beta \text {-induced OA chondrocytes } \\
\text { and collagenase-induced mouse } \\
\text { OA model }\end{array}$ & $\begin{array}{l}\text { Exosomal KLF3-AS1 promotes the } \\
\text { cartilage repair and chondrocyte } \\
\text { proliferation }\end{array}$ & - & $\begin{array}{l}\text { Liu et al. } \\
\text { (2018b) }\end{array}$ \\
\hline SHED-derived exosomes & MiR-100-5p & $\begin{array}{l}\text { Temporomandibular joint } \\
\text { chondrocytes }\end{array}$ & $\begin{array}{l}\text { SHED-exosomes suppresses the } \\
\text { inflammation in chondrocytes }\end{array}$ & mTOR & $\begin{array}{l}\text { Luo et al. } \\
\text { (2019) }\end{array}$ \\
\hline $\begin{array}{l}\text { Primary chondrocyte- } \\
\text { derived exosomes }\end{array}$ & MiR-95-5p & OA primary chondrocyte & $\begin{array}{l}\text { Promotes the cartilage formation and } \\
\text { prevents the } \mathrm{OA}\end{array}$ & $\mathrm{HDAC2} / 8$ & $\begin{array}{l}\text { Mao et al. } \\
\text { (2018a) }\end{array}$ \\
\hline MSC-derived exosomes & MiR-92a-3p & $\begin{array}{l}\text { Human MSC and OA primary } \\
\text { human chondrocytes }\end{array}$ & $\begin{array}{l}\text { Enhances the chondrogenesis and } \\
\text { suppresses the cartilage degradation via } \\
\text { targeting WNT5A }\end{array}$ & WNT5A & $\begin{array}{l}\text { Mao et al. } \\
(2018 b)\end{array}$ \\
\hline $\begin{array}{l}\text { Exosomes derived from OA } \\
\text { patient serum and LPS- } \\
\text { treated } \mathrm{C} 28 / \mathrm{l} 2 \text { cells }\end{array}$ & LncRNA PVT1 & $\begin{array}{l}\text { OA patient serum and LPS-induced } \\
\text { C28/12 cells }\end{array}$ & $\begin{array}{l}\text { Alleviates the lipopolysaccharide-induced } \\
\text { OA progression }\end{array}$ & $\begin{array}{l}\text { HMGB1/TLR4/NF- } \\
\text { кB pathway via mir- } \\
93-5 p\end{array}$ & $\begin{array}{l}\text { Meng et al. } \\
(2020)\end{array}$ \\
\hline $\begin{array}{l}\text { Exosomes derived from } \\
\text { osteoarthritic chondrocyte }\end{array}$ & MiR-449a-5p & $\begin{array}{l}\text { Chondrocytes and macrophages } \\
\text { of OA }\end{array}$ & $\begin{array}{l}\text { Enhances the mature } \mathrm{IL}-1 \beta \text { production of } \\
\text { macrophages and aggravates the } \\
\text { synovitis in } \mathrm{OA}\end{array}$ & ATG4B & Ni et al. (2019) \\
\hline $\begin{array}{l}\text { Exosomes derived from } \\
\text { curcumin-treated MSCs }\end{array}$ & $\begin{array}{l}\text { MiR-124 and } \\
\text { miR-143 }\end{array}$ & OA mouse models & $\begin{array}{l}\text { Curcumin reinforces the MSC-derived } \\
\text { exosomes in attenuating osteoarthritis }\end{array}$ & $\begin{array}{l}\text { NF-kB and ROCK1/ } \\
\text { TLR9 }\end{array}$ & $\begin{array}{l}\text { Qiu et al. } \\
\text { (2020) }\end{array}$ \\
\hline $\begin{array}{l}\text { Synovial MSC-derived } \\
\text { exosomes }\end{array}$ & MiR-129-5p & $\begin{array}{l}\text { OA patients and IL-1 } \beta \text {-induced } \\
\text { chondrocytes }\end{array}$ & Relieves the $\mathrm{IL}-1 \beta$ induced $\mathrm{OA}$ & HMGB1 & $\begin{array}{l}\text { Qiu et al. } \\
\text { (2021) }\end{array}$ \\
\hline $\begin{array}{l}\text { Synovial MSC-derived } \\
\text { exosomes }\end{array}$ & MiR-140-5p & Human synovial MSCs & $\begin{array}{l}\text { Enhances the cartilage tissue } \\
\text { regeneration and prevents the OA in a rat } \\
\text { model }\end{array}$ & RalA & $\begin{array}{l}\text { Tao et al. } \\
(2017)\end{array}$ \\
\hline $\begin{array}{l}\text { Exosomes derived from } \\
\text { synovial fibroblasts }\end{array}$ & LncRNA H19 & OA chondrocytes & $\begin{array}{l}\text { Promotes the chondrocyte proliferation } \\
\text { and migration and inhibits the degradation } \\
\text { of OA matrix }\end{array}$ & $\begin{array}{l}\text { MiR-106b-5p/ } \\
\text { TIMP2 }\end{array}$ & $\begin{array}{l}\text { Tan et al. } \\
(2020)\end{array}$ \\
\hline MSC-derived exosomes & MiR-135b & Chondrocyte of OA model rats & $\begin{array}{l}\text { TGF- } \beta 1 \text { promotes the chondrocyte } \\
\text { proliferation by regulating the Sp1 through } \\
\text { MSC-exosomes derived miR-135b }\end{array}$ & Sp1 & $\begin{array}{l}\text { Wang et al. } \\
\text { (2018) }\end{array}$ \\
\hline MSC-derived exosomes & MiR-135b & $\begin{array}{l}\text { Cartilage tissues and synovial } \\
\text { macrophages of model rats }\end{array}$ & $\begin{array}{l}\text { Attenuates the cartilage injury via } \\
\text { promoting the M2 synovial macrophage } \\
\text { polarization }\end{array}$ & MAPK6 & $\begin{array}{l}\text { Wang and Xu. } \\
\text { (2021) }\end{array}$ \\
\hline $\begin{array}{l}\text { Synovial MSC-derived } \\
\text { exosomes }\end{array}$ & MiR-155-5p & $\begin{array}{l}\text { OA chondrocytes and mouse } \\
\text { model of OA }\end{array}$ & $\begin{array}{l}\text { Prevents the OA via enhancing the } \\
\text { proliferation and migration, attenuating } \\
\text { the apoptosis, and modulating the ECM } \\
\text { secretion }\end{array}$ & Runx2 & $\begin{array}{l}\text { Wang Z. et al. } \\
(2021\end{array}$ \\
\hline $\begin{array}{l}\text { IPFP MSC-derived } \\
\text { exosomes }\end{array}$ & MiR-100-5p & IPFP of OA patients & $\begin{array}{l}\text { Protects the articular cartilage and } \\
\text { ameliorates the gait abnormalities via }\end{array}$ & 3'UTR of mTOR & $\begin{array}{l}\text { Wu et al. } \\
(2019)\end{array}$ \\
\hline
\end{tabular}


TABLE 1 | (Continued) Exosomes reported in the pathogenesis of OA

\begin{tabular}{|c|c|c|c|c|c|}
\hline $\begin{array}{l}\text { Classification of } \\
\text { exosomes }\end{array}$ & $\begin{array}{l}\text { Exosomal } \\
\text { inclusions }\end{array}$ & $\begin{array}{l}\text { Origin of } \\
\text { exosomes }\end{array}$ & Regulatory roles & Targets & References \\
\hline ADSC-derived exosomes & $\begin{array}{l}\text { MiR-145, } \\
\text { miR-221 }\end{array}$ & Chondrocytes of OA & $\begin{array}{l}\text { Promotes the chondrogenesis and } \\
\text { suppresses inflammation }\end{array}$ & Wnt/ $\beta$-catenin & $\begin{array}{l}\text { Zhao et al. } \\
(2020)\end{array}$ \\
\hline $\begin{array}{l}\text { Exosomes derived from } \\
\text { synovial fibroblasts }\end{array}$ & MiR-126-3p & Model rats of OA & $\begin{array}{l}\text { Suppresses the chondrocyte } \\
\text { inflammation and cartilage degradation }\end{array}$ & $\begin{array}{l}\text { IL-1 } \beta, \| \mathrm{L}-6 \text {, and } \\
\text { TNF- } \alpha\end{array}$ & $\begin{array}{l}\text { Zhou et al. } \\
\text { (2021) }\end{array}$ \\
\hline
\end{tabular}

\section{THE ROLES OF EXOSOMES IN THE TREATMENT OF OSTEOARTHRITIS}

Curcumin alleviates OA by enhancing MSC-derived exosomes. Curcumin significantly restores the expression of miR-143 and miR-124, and up-regulate the expression of NF-KB and ROCK1 in OA pathogenesis. The $3^{\prime}$ UTR of NF-KB and ROCK1 contains the binding sites of miR-143 and miR-124, respectively. Importantly, curcumin reduces the DNA methylation of miR143 and miR-124 promoters, suggesting that curcumin affects the methylation of these two miRNAs (Qiu et al., 2020).

Injection of bone marrow MSC-derived exosomes can alleviate the cartilage injury and pain in patients with OA. For example, exosomes treatment significantly attenuates the inhibitory effects of IL-1 $\beta$ on the chondrocyte proliferation and migration. Exosomes treatment significantly attenuates the IL- $1 \beta$-induced down-regulation of COL2A1 and ACAN and up-regulation of MMP13 and ADAMT5. Exosomes treatment significantly reduces the up-regulation of CGRP and iNOS in dorsal root ganglion (DRG) of OA model rats. Compared with untreated OA model rats, the paw withdrawal latency (PWL) value of exogenous $\mathrm{OA}$ model rats was significantly increased (Liu et al., 2019).

Cosenza et al. (2017) compared the roles of bone marrow MSC-derived exosomes and microbubbles/microparticles (MPs) in OA pathogenesis. In OA chondrocytes, bone marrow MSCderived exosomes and MPs both could inhibit the catabolism of MMP-13, ADAMTS5 and inflammatory marker iNOS, and reinduce the expression of aggrecan. Both exosomes and MPs could protect chondrocytes from apoptosis, inhibit the activation of macrophages, and protect model mice from joint injury. Exosomes and MPs replicate the main therapeutic effects of BM MSCs, suggesting that exosomes and MPs may contain the same substances that mediate intercellular communication.

In a bone defect treatment experiment, the osteochondral defect model was established in the trochlear groove of the distal femur of rats. One defect was treated with $100 \mu \mathrm{g}$ human extracellular exosomes, and the exosomes were injected into the joint after operation. Once a week for 12 weeks. Compared with control, the appearance of the defect was enhanced and the histological score was improved. At 12 weeks, the defects treated with exosomes showed complete recovery of cartilage and subchondral bone, which confirmed the effectiveness of human embryonic MSC-derived exosomes in cartilage repair (Zhang et al., 2016).

MiR-155-5p overexpression of synovial MSCs in vitro prevents the $\mathrm{OA}$ by reducing the chondrocyte apoptosis and regulating the extracellular matrix (ECM) secretion of chondrocytes. Studies have shown that synovial MSC-derived exosomes promote the proliferation and migration of $\mathrm{OA}$ chondrocytes and inhibit their apoptosis, but has no effect on the secretion of ECM. MiR-155-5p overexpression in exosomes show common characteristics and further promote the ECM secretion by targeting the Runx2. Exosomal miR-155-5p effectively prevent the pathological development of OA model mice. Furthermore, overexpression of Runx 2 partially reverses the effect of exosomal miR-155-5p on OA chondrocytes, proving that Runx2 is a direct target of miR-155-5p (Wang Z. et al., 2021).

Zhu et al. (2017) compared the efficacy of MSCs from synovial mesenchymal stem cells (SMMSC-Exos) and induced pluripotent stem cells (iMSC-Exos) in the treatment of OA. IMSC-Exos and SMMSC-Exos were injected into the joints of OA model mice induced by collagenase. The difference of therapeutic effects between them were evaluated by pathology, immunohistochemistry, cell count and scratch test. Both iMSC-Exos and SMMSC-Exos could reduce the symptoms of OA model in mice, but the therapeutic effects of iMSC-Exos were better than that of SMMSC-Exos, and iMSC-Exosomes promoted the migration and proliferation of chondrocytes more strongly. Since autologous iMSCs are inexhaustible in theory, iMSC-Exos may be a promising new method for the treatment of OA (Qiong et al., 2020).

\section{CONCLUSION AND PERSPECTIVES}

Exosomes are widely distributed in various body fluids, carrying and transmitting important signal molecules, forming a new intercellular information transmission system. Exosomes affect the physiological state of cells and are closely related to the occurrence and process of many diseases (Wang Y. et al., 2019). Almost all types of cells can secrete exosomes, and exosomes are also widely found in body fluids, including blood, tears, urine, saliva, milk, and ascites. At present, studies have found that exosomes contain the nucleic acids (miRNA, lncRNA, circRNA, mRNA, tRNA), protein, cholesterol, etc. The surface markers of exosomes are CD63, CD81, CD9, TSG101 and HSP70 (Li et al., 2018; Konečná et al., 2019). Therefore, exosomes can be associated with almost any disease and become an innovative hot spot in the research of disease biomarkers, disease mechanisms and drug development.

Exosomes transport proteins, mRNA, miRNA, IncRNA, circRNA, and even organelles into receptor cells, and participate in intercellular communication (Zhang et al., 2017). 
Exosomes play key roles in immune response, inflammation, angiogenesis, apoptosis, coagulation, waste disposal and other physiological processes. Exosomes from different cell sources contain different RNA and protein components, which can be used as early diagnostic biomarkers for a variety of diseases, and can also be used as carrier of drugs for disease treatment (Sung et al., 2018).

In addition, the characteristics of exosomes indicate its potential value in the treatment of OA. First, exosomes have a relatively long life span. Exosomes can be isolated from various body fluids and stored at $-80^{\circ} \mathrm{C}$ for a long time. Secondly, exosomes carry bioactive substances, including mRNAs, miRNAs, $\operatorname{lncRNAs}$ and proteins, to protect them from enzymatic degradation, which indicates that exosomes have the potential to deliver nucleic acid and protein drugs to target cells. Third, exosomes can be further modified to carry specific drugs to meet the needs of specific treatment regimens. In this review, we provide evidence of exosomes in the direct and indirect regulation of OA pathogenesis, with particular emphasis on the roles of miRNAs and lncRNAs (Colao et al., 2018; Yamashita et al., 2018).

However, the potential feasibility and targets of exosomes as OA treatment vectors are not fully understood, and the basic and clinical research still has a long way (Lakshmi et al., 2021; Stefanius et al., 2021). In the future, the following problems must be solved. First, exosomes will be isolated and purified to eliminate the interference of vesicles and other cell metabolites. The targets and mechanisms of exosomes in different tissues need to be clarified, and the efficacy and safety of exosomes in different

\section{REFERENCES}

Abramoff, B., and Caldera, F. E. (2020). Osteoarthritis. Med. Clin. North America 104 (2), 293-311. doi:10.1016/j.mcna.2019.10.007

Bai, J., Zhang, Y., Zheng, X., Huang, M., Cheng, W., Shan, H., et al. (2020). LncRNA MM2P-Induced, Exosome-Mediated Transfer of Sox9 from Monocyte-Derived Cells Modulates Primary Chondrocytes. Cell. Death Dis. 11 (9), 763. doi:10. 1038/s41419-020-02945-5

Barrett, T. J. (2020). Macrophages in Atherosclerosis Regression. Arterioscler Thromb Vasc. Biol. 40 (1), 20-33. doi:10.1161/ATVBAHA.119.312802

Beretti, F., Zavatti, M., Casciaro, F., Comitini, G., Franchi, F., Barbieri, V., et al. (2018). Amniotic Fluid Stem Cell Exosomes: Therapeutic Perspective. Biofactors. 44 (2), 158-167. doi:10.1002/biof.1407.Epub2018Jan16

Carlson, A. K., Rawle, R. A., Adams, E., Greenwood, M. C., Bothner, B., and June, R. K. (2018). Application of Global Metabolomic Profiling of Synovial Fluid for Osteoarthritis Biomarkers. Biochem. Biophys. Res. Commun. 499 (2), 182-188. doi:10.1016/j.bbrc.2018.03.117

Chang, Y.-H., Wu, K.-C., Harn, H.-J., Lin, S.-Z., and Ding, D.-C. (2018). Exosomes and Stem Cells in Degenerative Disease Diagnosis and Therapy. Cell. Transpl. 27 (3), 349-363. doi:10.1177/0963689717723636

Chen, B.-Y., Sung, C. W.-H., Chen, C., Cheng, C.-M., Lin, D. P.-C., Huang, C.-T., et al. (2019). Advances in Exosomes Technology. Clinica Chim. Acta. 493, 14-19. doi:10.1016/j.cca.2019.02.021

Chen, D., Gong, Y., Xu, L., Zhou, M., Li, J., Song, J., et al. (2019). Bidirectional Regulation of Osteogenic Differentiation by the FOXO Subfamily of Forkhead Transcription Factors in Mammalian MSCs. Cell. Prolif. 52 (2), e12540, doi:10. 1111/cpr.12540

Chen, X., Shi, Y., Xue, P., Ma, X., Li, J., and Zhang, J. (2020). Mesenchymal Stem Cell-Derived Exosomal microRNA-136-5p Inhibits Chondrocyte Degeneration in Traumatic Osteoarthritis by Targeting ELF3. Arthritis Res. Ther. 22 (1), 256. doi:10.1186/s13075-020-02325-6 animal models need to be evaluated. Furthermore, we should pay attention to the importance of vesicles secreted by cells. Vesicles are not only the stumbling blocks of exosomes research, but also the next research hotspot, because vesicles may play a more special role than exosomes.

\section{AUTHOR CONTRIBUTIONS}

$\mathrm{CM}$ was responsible for the design and writing of this review, including the roles of exosomes in the diagnosis of $\mathrm{OA}$ and the regulatory roles of exosomes in the pathogenesis of $\mathrm{OA}$. WZ was responsible for assisting $\mathrm{CM}$ in revising grammar. JF was responsible for assisting $\mathrm{CM}$ in writing about the roles of exosomes in OA diagnosis. XW was responsible for assisting in the design and writing of this review, funding the research group to carry out research in this field.

\section{FUNDING}

This project was supported by the National Science Foundation of China (No. 81302783), the Anhui Province Key Research and Development Plan (No. 1804a0802218), the Excellent talent project of Anhui Science and Technology University (No. XJYXRC201801), the special support plan of high-level talent introduction of Anhui University of Chinese Medicine (No. 2020rcZD001).

Clockaerts, S., Bastiaansen-Jenniskens, Y. M., Runhaar, J., Van Osch, G. J. V. M., Van Offel, J. F., VerhaarOffel., J. A. N., et al. (2010). The Infrapatellar Fat Pad Should Be Considered as an Active Osteoarthritic Joint Tissue: a Narrative Review. Osteoarthritis and Cartilage. 18 (7), 876-882. doi:10.1016/j.joca.2010. 03.014

Colao, I. L., Corteling, R., Bracewell, D., and Wall, I. (2018). Manufacturing Exosomes: A Promising Therapeutic Platform. Trends Mol. Med. 24 (3), 242-256. doi:10.1016/j.molmed.2018.01.006

Colombini, A., Perucca Orfei, C., Kouroupis, D., Ragni, E., De Luca, P., ViganÒ, M., et al. (2019). Mesenchymal Stem Cells in the Treatment of Articular Cartilage Degeneration: New Biological Insights for an OldTimer Cell. Cytotherapy. 21 (12), 1179-1197. doi:10.1016/j.jcyt.2019. 10.004

Cosenza, S., Ruiz, M., Toupet, K., Jorgensen, C., and Noël, D. (2017). Mesenchymal Stem Cells Derived Exosomes and Microparticles Protect Cartilage and Bone from Degradation in Osteoarthritis. Sci. Rep. 7 (1), 16214. doi:10.1038/s41598017-15376-8

Cui, D., Li, H., Xu, X., Ye, L., Zhou, X., Zheng, L., et al. (2017). Mesenchymal Stem Cells for Cartilage Regeneration of TMJ Osteoarthritis. Stem Cell Int. 2017, 1-11. doi:10.1155/2017/5979741

Domenis, R., Zanutel, R., Caponnetto, F., Toffoletto, B., Cifù, A., Pistis, C., et al. (2017). Characterization of the Proinflammatory Profile of Synovial FluidDerived Exosomes of Patients with Osteoarthritis. Mediators Inflamm. 2017, 1-11. doi:10.1155/2017/4814987

Dong, J., Li, L., Fang, X., and Zang, M. (2021). Exosome-Encapsulated microRNA127-3p Released from Bone Marrow-Derived Mesenchymal Stem Cells Alleviates Osteoarthritis through Regulating CDH11-Mediated Wnt/ ß-Catenin Pathway. J Pain Res. 14, 297-310. doi:10.2147/JPR.S291472

Duan, L., Liang, Y., Xu, X., Xiao, Y., and Wang, D. (2020). Recent Progress on the Role of miR-140 in Cartilage Matrix Remodelling and its Implications for Osteoarthritis Treatment. Arthritis Res. Ther. 22 (1), 194. doi:10.1186/s13075020-02290-0 
Familtseva, A., Jeremic, N., and Tyagi, S. C. (2019). Exosomes: Cell-Created Drug Delivery Systems. Mol. Cell. Biochem. 459 (1-2), 1-6. doi:10.1007/s11010-01903545-4

Gao, K., Zhu, W., Li, H., Ma, D., Liu, W., Yu, W., et al. (2020). Association between Cytokines and Exosomes in Synovial Fluid of Individuals with Knee Osteoarthritis. Mod. Rheumatol. 30 (4), 758-764. doi:10.1080/14397595. 2019.1651445

Geyer, M., and Schönfeld, C. (2018). Novel Insights into the Pathogenesis of Osteoarthritis. Curr. Rheumatol. Rev. 14 (2), 98-107. doi:10.2174/ 1573397113666170807122312

Gonda, A., Kabagwira, J., Senthil, G. N., and Wall, N. R. (2019). Internalization of Exosomes through Receptor-Mediated Endocytosis. Mol. Cancer Res. 17 (2), 337-347. doi:10.1158/1541-7786.MCR-18-0891

He, L., He, T., Xing, J., Zhou, Q., Fan, L., Liu, C., et al. (2020). Bone Marrow Mesenchymal Stem Cell-Derived Exosomes Protect Cartilage Damage and Relieve Knee Osteoarthritis Pain in a Rat Model of Osteoarthritis. Stem Cel. Res. Ther. 11 (1), 276. doi:10.1186/s13287-020-01781-w

Hunter, D. J., and Bierma-Zeinstra, S. (2019). Osteoarthritis. The Lancet. 393 (10182), 1745-1759. doi:10.1016/S0140-6736(19)30417-9

Jiang, L., Dong, H., Cao, H., Ji, X., Luan, S., and Liu, J. (2019). Exosomes in Pathogenesis, Diagnosis, and Treatment of Alzheimer's Disease. Med. Sci. Monit. 25, 3329-3335. doi:10.12659/MSM.914027

Jin, Z., Ren, J., and Qi, S. (2020a). Exosomal miR-9-5p Secreted by Bone MarrowDerived Mesenchymal Stem Cells Alleviates Osteoarthritis by Inhibiting Syndecan-1. Cell. Tissue Res. 381 (1), 99-114. doi:10.1007/s00441-02003193-x

Jin, Z., Ren, J., and Qi, S. (2020b). Human Bone Mesenchymal Stem Cells-Derived Exosomes Overexpressing microRNA-26a-5p Alleviate Osteoarthritis via Down-Regulation of PTGS2. Int. Immunopharmacol. 78, 105946. doi:10. 1016/j.intimp.2019.105946

Kalluri, R., and LeBleu, V. S. (2020). The Biology, Function, and Biomedical Applications of Exosomes. Science. 367 (6478), eaau6977, doi:10.1126/science. aau6977

Kan, H., Chan, P. K., Chan, P., Chiu, K., Yan, C., Yeung, S., et al. (2019). Nonsurgical Treatment of Knee Osteoarthritis. Hong Kong Med. J. 25 (2), 127-133. doi:10.12809/hkmj187600

Kim, M., Shin, D. I., Choi, B. H., and Min, B.-H. (2021). Exosomes from IL-1 $\beta-$ Primed Mesenchymal Stem Cells Inhibited IL-1 $\beta$ - and TNF- $\alpha$-Mediated Inflammatory Responses in Osteoarthritic SW982 Cells. Tissue Eng. Regen. Med. doi:10.1007/s13770-020-00324-x

Kim, S. W., Zhu, G. Q., and Bae, W. J. (2020). Mesenchymal Stem Cells Treatment for Erectile Dysfunction in Diabetic Rats. Sex. Med. Rev. 8 (1), 114-121. doi:10. 1016/j.sxmr.2019.09.003

Kolhe, R., Hunter, M., Liu, S., Jadeja, R. N., Pundkar, C., Mondal, A. K., et al. (2017). Gender-specific Differential Expression of Exosomal miRNA in Synovial Fluid of Patients with Osteoarthritis. Sci. Rep. 7 (1), 2029. doi:10. 1038/s41598-017-01905-y

Kolhe, R., Owens, V., Sharma, A., Lee, T. J., Zhi, W., Ghilzai, U., et al. (2020). SexSpecific Differences in Extracellular Vesicle Protein Cargo in Synovial Fluid of Patients with Osteoarthritis. Life (Basel). 10 (12), 337. doi:10.3390/life10120337

Konečná, B., Tóthová, Ľ., and Repiská, G. (2019). Exosomes-Associated DNA-New Marker in Pregnancy Complications? Int. J. Mol. Sci. 20 (12), 2890. doi:10.3390/ ijms 20122890

Lakshmi, S., Hughes, T. A., and Priya, S. (2021). Exosomes and Exosomal RNAs in Breast Cancer: A Status Update. Eur. J. Cancer. 144, 252-268. doi:10.1016/j.ejca. 2020.11.033

Li, J., Ding, Z., Li, Y., Wang, W., Wang, J., Yu, H., et al. (2020). BMSCs-Derived Exosomes Ameliorate Pain via Abrogation of Aberrant Nerve Invasion in Subchondral Bone in Lumbar Facet Joint Osteoarthritis. J. Orthop. Res. 38 (3), 670-679. doi:10.1002/jor.24497

Li, Z., Wang, Y., Xiao, K., Xiang, S., Li, Z., and Weng, X. (2018). Emerging Role of Exosomes in the Joint Diseases. Cell. Physiol. Biochem. 47 (5), 2008-2017. doi:10.1159/000491469

Liang, Y., Xu, X., Li, X., Xiong, J., Li, B., Duan, L., et al. (2020). ChondrocyteTargeted MicroRNA Delivery by Engineered Exosomes toward a Cell-free Osteoarthritis Therapy. ACS Appl. Mater. Inter. 12 (33), 36938-36947. doi:10. 1021/acsami.0c10458
Lin, T., Wu, N., Wang, L., Zhang, R., Pan, R., and Chen, Y.-F. (2021). Inhibition of Chondrocyte Apoptosis in a Rat Model of Osteoarthritis by Exosomes Derived from miR-140-5p-overexpressing Human Dental Pulp Stem Cells. Int. J. Mol. Med. 47 (3), 1. doi:10.3892/ijmm.2020.4840

Liu, C., Li, Y., Yang, Z., Zhou, Z., Lou, Z., and Zhang, Q. (2020). Kartogenin Enhances the Therapeutic Effect of Bone Marrow Mesenchymal Stem Cells Derived Exosomes in Cartilage Repair. Nanomedicine (Lond). 15 (3), 273-288. doi:10.2217/nnm-2019-0208

Liu, X., Wang, L., Ma, C., Wang, G., Zhang, Y., and Sun, S. (2019). Exosomes Derived from Platelet-Rich Plasma Present a Novel Potential in Alleviating Knee Osteoarthritis by Promoting Proliferation and Inhibiting Apoptosis of Chondrocyte via Wnt/ $\beta$-Catenin Signaling Pathway. J. Orthop. Surg. Res. 14 (1), 470. doi:10.1186/s13018-019-1529-7

Liu, Y., Lin, L., Zou, R., Wen, C., Wang, Z., and Lin, F. (2018a). MSC-derived Exosomes Promote Proliferation and Inhibit Apoptosis of Chondrocytes via IncRNA-KLF3-AS1/miR-206/GIT1 axis in Osteoarthritis. Cell Cycle. 17 (2122), 2411-2422. doi:10.1080/15384101.2018.1526603

Liu, Y., Zou, R., Wang, Z., Wen, C., Zhang, F., and Lin, F. (2018b). Exosomal KLF3AS1 from hMSCs Promoted Cartilage Repair and Chondrocyte Proliferation in Osteoarthritis. Biochem. J. 475 (22), 3629-3638. doi:10.1042/BCJ20180675

Luo, P., Jiang, C., Ji, P., Wang, M., and Xu, J. (2019). Exosomes of Stem Cells from Human Exfoliated Deciduous Teeth as an Anti-inflammatory Agent in Temporomandibular Joint Chondrocytes via miR-100-5p/mTOR. Stem Cel. Res. Ther. 10 (1), 216. doi:10.1186/s13287-019-1341-7

Mandl, L. A. (2019). Osteoarthritis Year in Review 2018: Clinical. Osteoarthritis and Cartilage. 27 (3), 359-364. doi:10.1016/j.joca.2018.11.001

Mao, G., Hu, S., Zhang, Z., Wu, P., Zhao, X., Lin, R., et al. (2018a). Exosomal miR95-5p Regulates Chondrogenesis and Cartilage Degradation via Histone Deacetylase 2/8. J. Cell. Mol. Med. 22 (11), 5354-5366. doi:10.1111/jcmm.13808

Mao, G., Zhang, Z., Hu, S., Zhang, Z., Chang, Z., Huang, Z., et al. (2018b). Exosomes Derived from miR-92a-3p-Overexpressing Human Mesenchymal Stem Cells Enhance Chondrogenesis and Suppress Cartilage Degradation via Targeting WNT5A. Stem Cell. Res. Ther. 9 (1), 247. doi:10.1186/s13287-0181004-0

Mashouri, L., Yousefi, H., Aref, A. R., Ahadi, A. m., Molaei, F., and Alahari, S. K. (2019). Exosomes: Composition, Biogenesis, and Mechanisms in Cancer Metastasis and Drug Resistance. Mol. Cancer. 18 (1), 75. doi:10.1186/ s12943-019-0991-5

Meldolesi, J. (2018). Exosomes and Ectosomes in Intercellular Communication. Curr. Biol. 28 (8), R435-R444. doi:10.1016/j.cub.2018.01.059

Meng, F., Li, Z., Zhang, Z., Yang, Z., Kang, Y., Zhao, X., et al. (2018). MicroRNA193b-3p Regulates Chondrogenesis and Chondrocyte Metabolism by Targeting HDAC3. Theranostics. 8 (10), 2862-2883. doi:10.7150/thno.23547

Meng, X.-M., Mak, T. S.-K., and Lan, H.-Y. (2019). Macrophages in Renal Fibrosis. Adv. Exp. Med. Biol. 1165, 285-303. doi:10.1007/978-981-13-8871-2_13

Meng, Y., Qiu, S., Sun, L., and Zuo, J. (2020). Knockdown of Exosome-mediated lnc-PVT1 Alleviates Lipopolysaccharide-induced Osteoarthritis Progression by Mediating the HMGB1/TLR4/NF-кB Pathway via miR-93-5p. Mol. Med. Rep. 22 (6), 5313-5325. doi:10.3892/mmr.2020.11594

Mianehsaz, E., Mirzaei, H. R., Mahjoubin-Tehran, M., Rezaee, A., Sahebnasagh, R., Pourhanifeh, M. H., et al. (2019). Mesenchymal Stem Cell-Derived Exosomes: a New Therapeutic Approach to Osteoarthritis? Stem Cel. Res. Ther. 10 (1), 340. doi:10.1186/s13287-019-1445-0

Ni, Z., Kuang, L., Chen, H., Xie, Y., Zhang, B., Ouyang, J., et al. (2019). The Exosome-like Vesicles from Osteoarthritic Chondrocyte Enhanced Mature IL$1 \beta$ Production of Macrophages and Aggravated Synovitis in Osteoarthritis. Cel. Death Dis. 10 (7), 522. doi:10.1038/s41419-019-1739-2

Niedermair, T., Lukas, C., Li, S., Stöckl, S., Craiovan, B., Brochhausen, C., et al. (2020). Influence of Extracellular Vesicles Isolated from Osteoblasts of Patients with Cox-Arthrosis And/or Osteoporosis on Metabolism and Osteogenic Differentiation of BMSCs. Front. Bioeng. Biotechnol. 8, 615520. doi:10.3389/ fbioe.2020.615520

O'Neill, T. W., and Felson, D. T. (2018). Mechanisms of Osteoarthritis (OA) Pain. Curr. Osteoporos. Rep. 16 (5), 611-616. doi:10.1007/s11914-018-0477-1

Otahal, A., Kramer, K., Kuten-Pella, O., Weiss, R., Stotter, C., Lacza, Z., et al. (2020). Characterization and Chondroprotective Effects of Extracellular Vesicles from Plasma- and Serum-Based Autologous Blood-Derived 
Products for Osteoarthritis Therapy. Front. Bioeng. Biotechnol. 8, 584050. doi:10.3389/fbioe.2020.584050

Qi, H., Liu, D.-P., Xiao, D.-W., Tian, D.-C., Su, Y.-W., and Jin, S.-F. (2019). Exosomes Derived from Mesenchymal Stem Cells Inhibit Mitochondrial Dysfunction-Induced Apoptosis of Chondrocytes via P38, ERK, and Akt Pathways. In Vitro Cell.Dev.Biol.-Animal. 55 (3), 203-210. doi:10.1007/ s11626-019-00330-x

Qiong, J., Xia, Z., Jing, L., and Haibin, W. (2020). Synovial Mesenchymal Stem Cells Effectively Alleviate Osteoarthritis through Promoting the Proliferation and Differentiation of Meniscus Chondrocytes. Eur. Rev. Med. Pharmacol. Sci. 24 (4), 1645-1655. doi:10.26355/eurrev_202002_20338

Qiu, B., Xu, X., Yi, P., and Hao, Y. (2020). Curcumin Reinforces MSC-derived Exosomes in Attenuating Osteoarthritis via Modulating the miR-124/NF-kB and miR-143/ROCK1/TLR9 Signalling Pathways. J. Cell. Mol. Med. 24 (18), 10855-10865. doi:10.1111/jcmm.15714

Qiu, M., Liu, D., and Fu, Q. (2021). MiR-129-5p Shuttled by Human Synovial Mesenchymal Stem Cell-Derived Exosomes Relieves IL-1 $\beta$ Induced Osteoarthritis via Targeting HMGB1. Life Sci. 269, 118987. doi:10.1016/j.lfs. 2020.118987

Russell, D. G., Huang, L., and VanderVen, B. C. (2019). Immunometabolism at the Interface between Macrophages and Pathogens. Nat. Rev. Immunol. 19 (5), 291-304. doi:10.1038/s41577-019-0124-9

Sacitharan, P. K. (2019). Ageing and Osteoarthritis. Subcell. Biochem. 91, 123-159. doi:10.1007/978-981-13-3681-2_6

Shan, S.-K., Lin, X., Li, F., Xu, F., Zhong, J.-Y., Guo, B., et al. (2020). Exosomes and Bone Disease. Curr Pharm Des. 25 (42), 4536-4549. doi:10.2174/ 1381612825666191127114054

Song, Y., Zhang, J., Xu, H., Lin, Z., Chang, H., Liu, W., et al. (2020). Mesenchymal Stem Cells in Knee Osteoarthritis Treatment: A Systematic Review and MetaAnalysis. J. Orthopaedic Translation. 24, 121-130. doi:10.1016/j.jot.2020. 03.015

Stefanius, K., Servage, K., and Orth, K. (2021). Exosomes in Cancer Development. Curr. Opin. Genet. Dev. 66, 83-92. doi:10.1016/j.gde.2020.12.018

Sun, H., Hu, S., Zhang, Z., Lun, J., Liao, W., and Zhang, Z. (2019). Expression of Exosomal microRNAs during Chondrogenic Differentiation of Human Bone Mesenchymal Stem Cells. J. Cel. Biochem. 120 (1), 171-181. doi:10.1002/jcb. 27289

Sun, Y., Kang, S., Pei, S., Sang, C., and Huang, Y. (2020). MiR93-5p Inhibits Chondrocyte Apoptosis in Osteoarthritis by Targeting lncRNA CASC2. BMC. Musculoskelet. Disord. 21 (1), 26. doi:10.1186/s12891-019-3025-y

Sung, S., Kim, J., and Jung, Y. (2018). Liver-Derived Exosomes and Their Implications in Liver Pathobiology. Int J Mol Sci. 19 (12), 3715. doi:10.3390/ ijms19123715

Tan, F., Wang, D., and Yuan, Z. (2020). The Fibroblast-like Synoviocyte Derived Exosomal Long Non-coding RNA H19 Alleviates Osteoarthritis Progression through the miR-106b-5p/TIMP2 Axis. Inflammation. 43 (4), 1498-1509. doi:10.1007/s10753-020-01227-8

Tao, S.-C., Yuan, T., Zhang, Y.-L., Yin, W.-J., Guo, S.-C., and Zhang, C.-Q. (2017). Exosomes Derived from miR-140-5p-Overexpressing Human Synovial Mesenchymal Stem Cells Enhance Cartilage Tissue Regeneration and Prevent Osteoarthritis of the Knee in a Rat Model. Theranostics. 7 (1), 180-195. doi:10.7150/thno.17133

Tofiño-Vian, M., Guillén, M. I., Pérez del Caz, M. D., Caz, M. D., Castejón, M. A., and Alcaraz, M. J. (2017). Extracellular Vesicles from Adipose-Derived Mesenchymal Stem Cells Downregulate Senescence Features in Osteoarthritic Osteoblasts. Oxid. Med. Cell Longevity. 2017, 1-12. doi:10. 1155/2017/7197598

Toh, W. S., Lai, R. C., Hui, J. H. P., and Lim, S. K. (2017). MSC Exosome as a Cellfree MSC Therapy for Cartilage Regeneration: Implications for Osteoarthritis Treatment. Semin. Cel Developmental Biol. 67, 56-64. doi:10.1016/j.semcdb. 2016.11.008

Wang, R., Jiang, W., Zhang, L., Xie, S., Zhang, S., Yuan, S., et al. (2020). Intraarticular Delivery of Extracellular Vesicles Secreted by Chondrogenic Progenitor Cells from MRL/MpJ Superhealer Mice Enhances Articular Cartilage Repair in a Mouse Injury Model. Stem Cel. Res. Ther. 11 (1), 93. doi:10.1186/s13287-020-01594-x

Wang, R., and Xu, B. (2021). TGF- $\beta 1$-modified MSC-Derived Exosomal miR-135b Attenuates Cartilage Injury via Promoting M2 Synovial Macrophage
Polarization by Targeting MAPK6. Cell Tissue Res. 384, 113-127. doi:10. 1007/s00441-020-03319-1

Wang, R., Xu, B., and Xu, H. (2018). TGF- $\beta 1$ Promoted Chondrocyte Proliferation by Regulating Sp1 through MSC-Exosomes Derived miR-135b. Cell Cycle. 17 (24), 2756-2765. doi:10.1080/15384101.2018.1556063

Wang, S., Liu, R., Yu, Q., Dong, L., Bi, Y., and Liu, G. (2019). Metabolic Reprogramming of Macrophages during Infections and Cancer. Cancer Lett. 452, 14-22. doi:10.1016/j.canlet.2019.03.015

Wang, Y., He, S. H., Liang, X., Zhang, X. X., Li, S. S., and Li, T. F. (2021). ATF4 -modified Serum Exosomes Derived from Osteoarthritic Mice Inhibit Osteoarthritis by Inducing Autophagy. IUBMB. Life. 73 (1), 146-158. doi:10.1002/iub.2414

Wang, Y., Xie, Y., Zhang, A., Wang, M., Fang, Z., and Zhang, J. (2019). Exosomes: An Emerging Factor in Atherosclerosis. Biomed. Pharmacother. 115, 108951. doi:10.1016/j.biopha.2019.108951

Wang, Y., Yu, D., Liu, Z., Zhou, F., Dai, J., Wu, B., et al. (2017). Exosomes from Embryonic Mesenchymal Stem Cells Alleviate Osteoarthritis through Balancing Synthesis and Degradation of Cartilage Extracellular Matrix. Stem Cel. Res. Ther. 8 (1), 189. doi:10.1186/s13287-017-0632-0

Wang, Z., Yan, K., Ge, G., Zhang, D., Bai, J., Guo, X., et al. (2021). Exosomes Derived from miR-155-5p-Overexpressing Synovial Mesenchymal Stem Cells Prevent Osteoarthritis via Enhancing Proliferation and Migration, Attenuating Apoptosis, and Modulating Extracellular Matrix Secretion in Chondrocytes. Cell. Biol. Toxicol. 37 (1), 85-96. doi:10.1007/s10565-020-09559-9

Wu, J., Kuang, L., Chen, C., Yang, J., Zeng, W.-N., Li, T., et al. (2019). miR-100-5pabundant Exosomes Derived from Infrapatellar Fat Pad MSCs Protect Articular Cartilage and Ameliorate Gait Abnormalities via Inhibition of mTOR in Osteoarthritis. Biomaterials. 206, 87-100. doi:10.1016/j.biomaterials.2019. 03.022

Xu, X., Liang, Y., Li, X., Ouyang, K., Wang, M., Cao, T., et al. (2021). Exosomemediated Delivery of Kartogenin for Chondrogenesis of Synovial Fluid-Derived Mesenchymal Stem Cells and Cartilage Regeneration. Biomaterials. 269, 120539. doi:10.1016/j.biomaterials.2020.120539

Xue, H., Tu, Y., Ma, T., Wen, T., Yang, T., Xue, L., et al. (2019). miR-93-5p Attenuates IL-1 $\beta$-induced Chondrocyte Apoptosis and Cartilage Degradation in Osteoarthritis Partially by Targeting TCF4. Bone. 123, 129-136. doi:10.1016/ j.bone.2019.03.035

Yamashita, T., Takahashi, Y., and Takakura, Y. (2018). Possibility of ExosomeBased Therapeutics and Challenges in Production of Exosomes Eligible for Therapeutic Application. Biol. Pharm. Bull. 41 (6), 835-842. doi:10.1248/bpb. b18-00133

Yang, R.-Z., Zheng, H.-L., Xu, W.-N., Zheng, X.-F., Li, B., Jiang, L.-S., et al. (2021). Vascular Endothelial Cell-Secreted Exosomes Facilitate Osteoarthritis Pathogenesis by Promoting Chondrocyte Apoptosis. Aging (Albany NY). 13 (3), 4647-4662. doi:10.18632/aging.202506

Yuan, Z., Li, Q., Luo, S., Liu, Z., Luo, D., Zhang, B., et al. (2016). PPAR $\gamma$; and Wnt Signaling in Adipogenic and Osteogenic Differentiation of Mesenchymal Stem Cells. Curr Stem Cell Res Ther. 11 (3), 216-225. doi:10.2174/ $1574888 \times 10666150519093429$

Zavatti, M., Beretti, F., Casciaro, F., Bertucci, E., and Maraldi, T. (2020). Comparison of the Therapeutic Effect of Amniotic Fluid Stem Cells and Their Exosomes on Monoiodoacetate-induced Animal Model of Osteoarthritis. Biofactors. 46 (1), 106-117. doi:10.1002/biof.1576

Zhang, J., Rong, Y., Luo, C., and Cui, W. (2020). Bone Marrow Mesenchymal Stem Cell-Derived Exosomes Prevent Osteoarthritis by Regulating Synovial Macrophage Polarization. Aging(Albany NY). 12 (24), 25138-25152. doi:10. 18632/aging. 104110

Zhang, L., and Yu, D. (2019). Exosomes in Cancer Development, Metastasis, and Immunity. Biochim. Biophys. Acta (Bba) - Rev. Cancer. 1871 (2), 455-468. doi:10.1016/j.bbcan.2019.04.004

Zhang, S., Chu, W. C., Lai, R. C., Lim, S. K., Hui, J. H. P., and Toh, W. S. (2016). Exosomes Derived from Human Embryonic Mesenchymal Stem Cells Promote Osteochondral Regeneration. Osteoarthritis Cartilage. 24 (12), 2135-2140. doi:10.1016/j.joca.2016.06.022

Zhang, S., Teo, K. Y. W., Chuah, S. J., Lai, R. C., Lim, S. K., and Toh, W. S. (2019). MSC Exosomes Alleviate Temporomandibular Joint Osteoarthritis by Attenuating Inflammation and Restoring Matrix Homeostasis. Biomaterials. 200, 35-47. doi:10.1016/j.biomaterials.2019.02.006 
Zhang, Y., Hu, Y.-W., Zheng, L., and Wang, Q. (2017). Characteristics and Roles of Exosomes in Cardiovascular Disease. DNA Cel Biol. 36 (3), 202-211. doi:10. 1089/dna.2016.3496

Zhao, C., Chen, J. Y., Peng, W. M., Yuan, B., Bi, Q., and Xu, Y. J. (2020). Exosomes from Adipose-derived Stem Cells Promote Chondrogenesis and Suppress Inflammation by Upregulating miR-145 and miR-221. Mol. Med. Rep. 21 (4), 1881-1889. doi:10.3892/mmr.2020.10982

Zhao, P., Xiao, L., Peng, J., Qian, Y. Q., and Huang, C. C. (2018). Exosomes Derived from Bone Marrow Mesenchymal Stem Cells Improve Osteoporosis through Promoting Osteoblast Proliferation via MAPK Pathway. Eur. Rev. Med. Pharmacol. Sci. 22 (12), 3962-3970. doi:10.26355/eurrev_201806_15280

Zhao, Y., and Xu, J. (2018). Synovial Fluid-Derived Exosomal lncRNA PCGEM1 as Biomarker for the Different Stages of Osteoarthritis. Int. Orthopaedics (Sicot). 42 (12), 2865-2872. doi:10.1007/s00264-018-4093-6

Zheng, L., Wang, Y., Qiu, P., Xia, C., Fang, Y., Mei, S., et al. (2019). Primary Chondrocyte Exosomes Mediate Osteoarthritis Progression by Regulating Mitochondrion and Immune Reactivity. Nanomedicine. 14 (24), 3193-3212. doi:10.2217/nnm-2018-0498

Zhou, Q.-F., Cai, Y.-z., and Lin, X.-j. (2020). The Dual Character of Exosomes in Osteoarthritis: Antagonists and Therapeutic Agents. Acta Biomater. 105, 15-25. doi:10.1016/j.actbio.2020.01.040

Zhou, X., Liang, H., Hu, X., An, J., Ding, S., Yu, S., et al. (2020). BMSC-derived Exosomes from Congenital Polydactyly Tissue Alleviate Osteoarthritis by
Promoting Chondrocyte Proliferation. Cell Death Discov. 6 (1), 142. doi:10. 1038/s41420-020-00374-Z

Zhou, Y., Ming, J., Li, Y., Li, B., Deng, M., Ma, Y., et al. (2021). Exosomes Derived from miR-126-3p-Overexpressing Synovial Fibroblasts Suppress Chondrocyte Inflammation and Cartilage Degradation in a Rat Model of Osteoarthritis. $\mathrm{Cel}$ Death Discov. 7 (1), 37. doi:10.1038/s41420-021-00418-y

Zhu, Y., Wang, Y., Zhao, B., Niu, X., Hu, B., Li, Q., et al. (2017). Comparison of Exosomes Secreted by Induced Pluripotent Stem Cell-Derived Mesenchymal Stem Cells and Synovial Membrane-Derived Mesenchymal Stem Cells for the Treatment of Osteoarthritis. Stem Cel. Res. Ther. 8 (1), 64. doi:10.1186/s13287017-0510-9

Conflict of Interest: The authors declare that the research was conducted in the absence of any commercial or financial relationships that could be construed as a potential conflict of interest.

Copyright (c) 2021 Miao, Zhou, Wang and Fang. This is an open-access article distributed under the terms of the Creative Commons Attribution License (CC BY). The use, distribution or reproduction in other forums is permitted, provided the original author(s) and the copyright owner(s) are credited and that the original publication in this journal is cited, in accordance with accepted academic practice. No use, distribution or reproduction is permitted which does not comply with these terms. 